\title{
Radiative transfer theory verified by controlled laboratory experiments
}

\author{
Michael I. Mishchenko, ${ }^{1, *}$ Dennis H. Goldstein, ${ }^{2}$ Jacek Chowdhary, ${ }^{3}$ and Arthur Lompado ${ }^{2}$ \\ ${ }^{1}$ NASA Goddard Institute for Space Studies, 2880 Broadway, New York, New York 10025, USA \\ ${ }^{2}$ Polaris Sensor Technologies, 200 West Court Sq., Huntsville, Alabama 35801, USA \\ ${ }^{3}$ Department of Applied Physics and Applied Mathematics, Columbia University, 2880 Broadway, New York 10025, USA \\ *Corresponding author: michael.i.mishchenko@nasa.gov
}

Received June 7, 2013; revised July 19, 2013; accepted August 2, 2013;

posted August 7, 2013 (Doc. ID 191920); published September 5, 2013

\begin{abstract}
We report the results of high-accuracy controlled laboratory measurements of the Stokes reflection matrix for suspensions of submicrometer-sized latex particles in water and compare them with the results of a numerically exact computer solution of the vector radiative transfer equation (VRTE). The quantitative performance of the VRTE is monitored by increasing the volume packing density of the latex particles from $2 \%$ to $10 \%$. Our results indicate that the VRTE can be applied safely to random particulate media with packing densities up to 2\%. VRTE results for packing densities of the order of $5 \%$ should be taken with caution, whereas the polarized bidirectional reflectivity of suspensions with larger packing densities cannot be accurately predicted. We demonstrate that a simple modification of the phase matrix entering the VRTE based on the so-called static structure factor can be a promising remedy that deserves further examination.

OCIS codes: (030.5620) Radiative transfer; (290.4210) Multiple scattering; (290.5820) Scattering measurements; (290.5850) Scattering, particles; (290.5855) Scattering, polarization.

http://dx.doi.org/10.1364/OL.38.003522
\end{abstract}

Despite its extensive use in numerous areas of science and engineering for more than a century, the radiative transfer equation (RTE) had not had a solid physical foundation until quite recently. The derivation of the RTE for sparse discrete random media directly from the Maxwell equations [1,2] has finally made the RT theory (RTT) a legitimate branch of physical optics. Yet some of the assumptions used in this derivation are still formulated qualitatively rather than quantitatively. Perhaps the most important assumption is the limit of vanishing volume packing density of the particles populating the scattering medium: $\rho \rightarrow 0$. There has been some progress in quantifying this limit based on comparisons of $\rho$ independent RTT calculations with direct computer solutions of the Maxwell equations for random multiparticle groups with substantial packing densities [3-7]. However, such comparisons are still impracticable for realistic media composed of extremely large numbers of particles, e.g., particle suspensions and powder surfaces. This makes controlled laboratory experiments an essential tool for determining the maximal allowable packing density $\rho_{\max }$ defined such that for $\rho<\rho_{\max }$ the RTT predictions can be expected to be quantitatively accurate.

In this Letter, we report the results of controlled laboratory measurements of the Stokes reflection matrix for suspensions of microscopic latex particles in water and compare them with the results of numerically exact computer solutions of the vector RTE. By increasing the volume packing density of the latex particles, we monitor the quantitative performance of the RTE and establish its range of applicability. We then discuss a simple "patch" that could be used to extend this range to larger packing densities.

Note that the use of particle suspensions in this study was advantageous for two key reasons. First, it allowed us to vary the particle packing density in a controllable way (cf. $[8,9])$. Second, random Brownian motion of particles during the measurement ensured the compliance of the scattering sample with the requirement of ergodicity necessary for the derivation of the RTE [1].

The scattering geometry shown schematically in Fig. 1 was designed to simplify the numerical solution of the RTE by making applicable the assumption of a laterally infinite plane-parallel scattering layer. Specifically, the scattering sample was an aqueous suspension of nearly monodisperse latex spheres filling a $25.4 \mathrm{~mm}$ diameter cylindrical cavity of $2 \mathrm{~mm}$ depth with absorptive side walls and bottom surface. The sample was illuminated by a parallel beam of light with a diameter exceeding that of the cavity, while the receiving optics sampled a small $2 \mathrm{~mm}$ diameter area near the sample's center, as shown by the inset in Fig. 2 .

The nadir angle $\theta_{0}$ of the incidence direction was kept constant at $20^{\circ}$. All scattering measurements were taken in the vertical plane through the incidence direction and were limited to the following zenith angles $\theta$ of the reflected light: $-60^{\circ},-50^{\circ},-45^{\circ},-5^{\circ}, 5^{\circ}, 30^{\circ}, 45^{\circ}$, and $60^{\circ}$; the sign convention is explained in Fig. 1.

In the standard framework of plane-parallel scattering geometry, the polarized reflectivity of the sample is fully described by the $4 \times 4$ reflection matrix $\mathbf{R}\left(\theta, \theta_{0}\right)$ specifying the transformation of the Stokes parameters of the

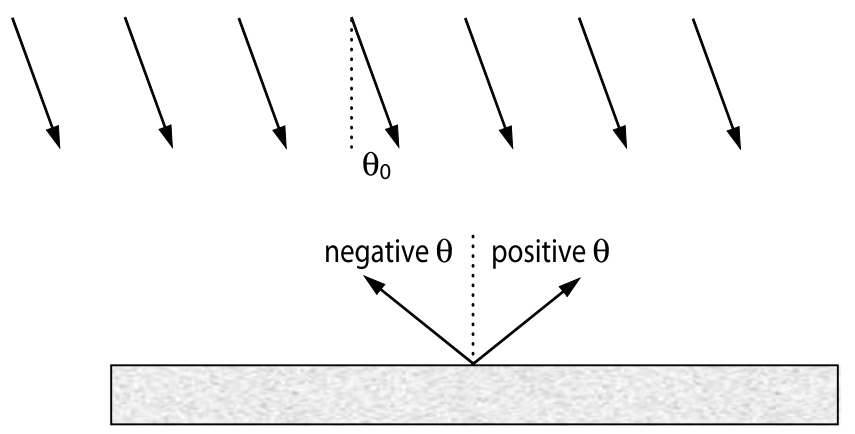

Fig. 1. Scattering geometry. 
incident beam into those of the reflected light [1]. The laboratory multidirectional spectro-polarimeter described in [10] was partially reconfigured to accommodate an open, horizontally oriented liquid sample. This instrument allows one to measure with high accuracy the ratios $R_{i j}\left(\theta, \theta_{0}\right) / R_{11}\left(\theta, \theta_{0}\right)$ over a broad and quasicontinuous range of visible and near-infrared wavelengths, where $i, j=1, \ldots, 4$. For the purposes of this study, the measurements were averaged over two $10 \mathrm{~nm}$ wide intervals centered at the wavelengths 595 and $695 \mathrm{~nm}$. In agreement with theory, the elements of the upper-right and lower-left $2 \times 2$ submatrices of the reflection matrix were found to be essentially zero. The residual deviations of these elements from the theoretical value zero were indicative of $\sim 1 \%$ measurement uncertainties in the ratios $R_{i j} / R_{11}$. Therefore, the latter will be indicated by $\pm 1 \%$ error bars in the forthcoming plots comparing theory and experiment.

According to the manufacturer (Bangs Laboratories, Inc.), the average radius of the latex microspheres was close to $350 \mathrm{~nm}$, while their refractive index relative to that of water is known to be close to 1.196 at the two selected wavelengths. The measurements of the ratios $R_{i j} / R_{11}$ were performed for three increasing values of the volume packing density: $\rho=2 \%, 5 \%$, and $10 \%$. Figure 2 shows a representative subset of the results thus obtained. Given the independent detailed knowledge of the scattering sample's macro and microphysical
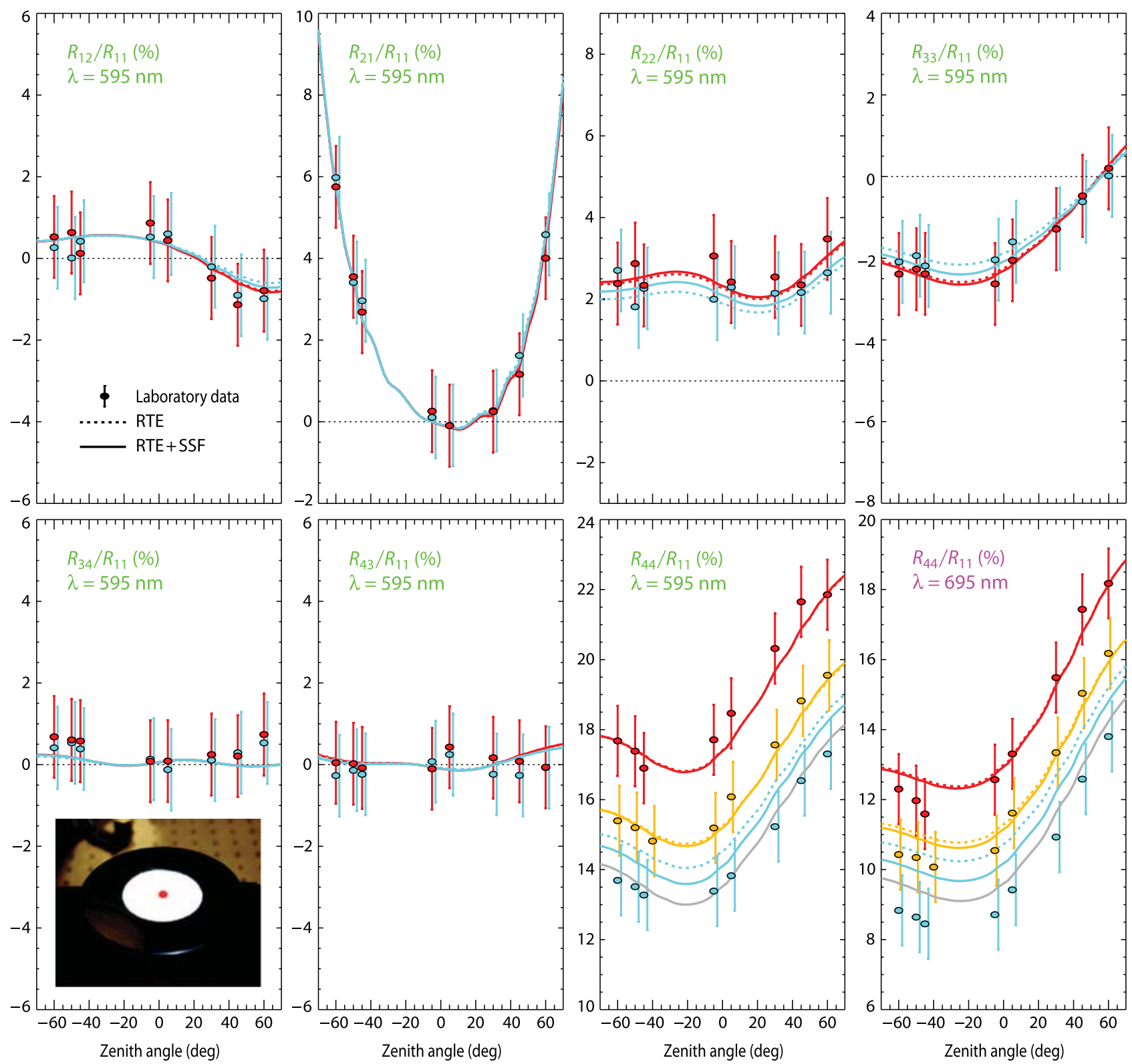

Fig. 2. Measurements (filled circles), RTT computations (dashed curves), and RTT+SSF computations (solid curves) of the ratios of the elements of the Stokes reflection matrix $\mathbf{R}$ for aqueous suspensions of latex particles with packing densities $\rho=2 \%$ (red color), 5\% (yellow color), and 10\% (blue color). The two gray curves show the best fit of RTT + SSF theoretical results for $\rho=15 \%$ to the laboratory data for $\rho=10 \%$. The inset is a photograph of the scattering sample in which the red dot shows the size and approximate location of the area sampled by the receiving optics. 
parameters, this dataset constituted an excellent quantitative test of the RTT.

To solve the vector RTE for the plane-parallel geometry, we used the well-known adding/doubling method $[1,11]$ in which the reflection and transmission matrices of the water-air interface were incorporated according to the Kirchhoff approximation [12], otherwise known as the ray-optics approximation [1] $1 \overline{3}$. Based on extensive calculations, we found that below a certain nontrivial surface roughness threshold, the cumulative reflection matrix $\mathbf{R}\left(\theta, \theta_{0}\right)$ of the entire system becomes essentially independent of the surface roughness scale except at zenith angles $\theta$ extremely close to the specular reflection direction. This implies that microscopic undulations of the water surface that could be caused by air pressure fluctuations and/or mechanical vibrations of the experimental setup had negligible effect on our measurements and calculations for the specific sampled scattering directions. The single-scattering quantities entering the RTE were computed according to the standard LorenzMie theory [14].

Instead of using the manufacturer-specified characteristics of the latex-particle suspensions, we considered them unknown and "retrieved" them by looking for the best fit of theoretical RTE results to the laboratory data at both selected wavelengths. We found that the most difficult task was to fit the measured ratio $R_{44} / R_{11}$. This necessitated, for example, the use of an extremely narrow size distribution of latex particles. Nevertheless, as Fig. 2 demonstrates, a near-perfect fit was obtained in the case of the $2 \%$ suspension for the following values of the model parameters: effective radius of the size distribution $350 \mathrm{~nm}$, effective variance of the size distribution 0.001 , refractive index 1.196, and optical thickness 128 at $595 \mathrm{~nm}$. All these values are in excellent quantitative agreement with the independently available information. Importantly, no satisfactory fit was found for effective radii and refractive indices deviating by more than $\pm 15 \mathrm{~nm}$ and \pm 0.04 from the corresponding best-fit values.

Obtaining a good theoretical fit to the measurement results for the 5\% suspension was also possible and resulted in the same values of the microphysical latexparticle parameters (see the two $R_{44} / R_{11}$ panels in Fig. 2). However, the corresponding optical thickness value 485 at $595 \mathrm{~nm}$ was significantly greater than 2.5 times that for the $2 \%$ suspension. Fitting the laboratory data for the $10 \%$ suspension turned out to be impossible even when the model optical thickness was increased to infinity.

To improve the fits for the $5 \%$ and $10 \%$ suspensions, we modified the phase matrix entering the classical vector RTE by applying a simple phenomenological patch based on the so-called static structure factor (SSF) $[\underline{15}, \underline{16}]$. This phenomenological recipe is intended to partially account for mutual correlations of particle positions in a densely packed discrete random medium and serves to make $\rho$ an explicit model parameter. The SSF was computed using the Percus-Yevick approximation for impenetrable monodisperse spheres [17].

Figure 2 shows that despite its technical simplicity, the introduction of the SSF appears to be a promising modeling tool. Although the fit remained virtually the same for the $2 \%$ and $5 \%$ suspensions, the scaling of the optical thickness became more realistic (115 versus 280). The solid blue $R_{44} / R_{11}$ curve at $595 \mathrm{~nm}$ is now completely within the measurement error bars for an optical thickness of 550, and the corresponding fit at $695 \mathrm{~nm}$ was substantially improved.

Interestingly, a nearly perfect fit to the laboratory data for the suspension with the $10 \%$ packing density can be obtained with RTT + SSF computations for $\rho=15 \%$, as the two gray curves in Fig. 2 demonstrate. This result suggests that the RTT + SSF patch may necessitate values of the formal packing-density parameter somewhat larger than the actual ones to yield an improved fit to the results of bidirectional photo-polarimetric measurements.

In summary, our results further corroborate the methodology used in the microphysical derivation of the vector RTE from the macroscopic Maxwell equations [1,2] and suggest that the RTE can be applied safely to random particulate media with packing densities up to $2 \%$. RTE results for packing densities of the order of $5 \%$ should be taken with caution, whereas larger packing densities cannot be accurately handled and definitely require the use of alternative modeling tools [18]. In this regard, the simple modification of the phase matrix based on the SSF shows certain promise and should be further analyzed. Given the widespread (and often uncritical) use of the RTE in many science and engineering disciplines (e.g., [19-25] and references therein), the results of our study should provide important guidance in the process of identifying appropriate modeling tools and deciding whether the RTE can even be used depending on the specific practical problem in hand.

We appreciate useful discussions with Brian Cairns, David Chenault, Thomas Kulp, Li Liu, and Thomas Reichardt. This material is based upon work supported by the Department of Energy under Award Number DE-SC0008285 managed by Victoria Franques, the NASA Remote Sensing Theory Program managed by Lucia Tsaoussi, and the NASA Radiation Sciences Program managed by Hal Maring. This report was prepared as an account of work sponsored by an agency of the United States Government. Neither the United States Government nor any agency thereof, nor any of their employees, makes any warranty, express or implied, or assumes any legal liability or responsibility for the accuracy, completeness, or usefulness of any information, apparatus, product, or process disclosed, or represents that its use would not infringe privately owned rights. Reference herein to any specific commercial product, process, or service by trade name, trademark, manufacturer, or otherwise does not necessarily constitute or imply its endorsement, recommendation, or favoring by the United States Government or any agency thereof. The views and opinions of authors expressed herein do not necessarily state or reflect those of the United States Government or any agency thereof.

\section{References}

1. M. I. Mishchenko, L. D. Travis, and A. A. Lacis, Multiple Scattering of Light by Particles (Cambridge, 2006), http://www.giss.nasa.gov/staff/mmishchenko/books.html.

2. M. I. Mishchenko, Opt. Express 18, 19770 (2010).

3. M. I. Mishchenko, L. Liu, D. W. Mackowski, B. Cairns, and G. Videen, Opt. Express 15, 2822 (2007). 
4. F. Voit, J. Schäfer, and A. Kienle, Opt. Lett. 34, 2593 (2009).

5. J. M. Dlugach, M. I. Mishchenko, L. Liu, and D. W. Mackowski, J. Quant. Spectrosc. Radiat. Transfer 112, 2068 (2011).

6. K. Muinonen, M. I. Mishchenko, J. M. Dlugach, E. Zubko, A. Penttilä, and G. Videen, Astrophys. J. 760, 118 (2012)

7. D. W. Mackowski and M. I. Mishchenko, J. Quant. Spectrosc. Radiat. Transfer 123, 103 (2013).

8. M. J. Raković, G. W. Kattawar, M. Mehrúbeoğlu, B. D. Cameron, L. V. Wang, S. Rastegar, and G. L. Coté, Appl. Opt. 38, 3399 (1999).

9. N. Riviere, R. Ceolato, and L. Hespel, "Polarimetric and angular light scattering from dense media: comparison of a vectorial radiative transfer model with analytical, stochastic and experimental approaches," J. Quant. Spectrosc. Radiat. Transfer (to be published).

10. D. H. Goldstein, R. A. Chipman, and D. B. Chenault, Opt. Eng. 28, 282120 (1989).

11. J. W. Hovenier, C. van der Mee, and H. Domke, Transfer of Polarized Light in Planetary Atmospheres (Kluwer, 2004).

12. L. Tsang, J. A. Kong, and R. T. Shin, Theory of Microwave Remote Sensing (Wiley, 1985).

13. J. Chowdhary, B. Cairns, F. Waquet, K. Knobelspiesse, M. Ottaviani, J. Redemann, L. Travis, and M. Mishchenko, Remote Sens. Environ. 118, 284 (2012).
14. M. I. Mishchenko, L. D. Travis, and A. A. Lacis, Scattering, Absorption, and Emission of Light by Small Particles (Cambridge University, 2002), http://www.giss.nasa.gov/ staff/mmishchenko/books.html.

15. P. E. Wolf, G. Maret, E. Akkermans, and R. Maynard, J. Phys. France 49, 63 (1988).

16. M. I. Mishchenko, J. Quant. Spectrosc. Radiat. Transfer 52, 95 (1994)

17. R. Balescu, Equilibrium and Nonequilibrium Statistical Mechanics (Wiley, 1975).

18. V. P. Tishkovets, E. V. Petrova, and M. I. Mishchenko, J. Quant. Spectrosc. Radiat. Transfer 112, 2095 (2011).

19. G. L. Stephens, Remote Sensing of the Lower Atmosphere (Oxford University, 1994).

20. K. N. Liou, An Introduction to Atmospheric Radiation (Academic, 2002).

21. V. V. Tuchin, L. V. Wang, and D. A. Zimnyakov, Optical Polarization in Biomedical Applications (Springer, 2006).

22. M. I. Mishchenko, V. K. Rosenbush, N. N. Kiselev, D. F. Lupishko, V. P. Tishkovets, V. G. Kaydash, I. N. Belskaya, Y. S. Efimov, and N. M. Shakhovskoy, Polarimetric Remote Sensing of Solar System Objects (Akademperiodyka, 2010), arXiv: 1010.1171

23. J. R. Howell, R. Siegel, and M. P. Mengüç, Thermal Radiation Heat Transfer (CRC Press, 2011).

24. M. Wendisch and P. Yang, Theory of Atmospheric Radiative Transfer (Wiley, 2012).

25. M. Modest, Radiative Heat Transfer (Academic, 2013). 